\title{
Morphological evaluation of the protective role of dark soy sauce against acrylamide induced neurotoxicity in albino rats
}

\author{
H.F. Abdelall ${ }^{1}$, T.A. AlGhamrawy ${ }^{2}$, D. Helmy ${ }^{1}$ \\ ${ }^{1}$ Department of Histology, Faculty of Medicine, Cairo University, Cairo, Egypt \\ ${ }^{2}$ Department of Anatomy, Faculty of Medicine, Cairo University, Cairo, Egypt \\ [Received 8 June 2014; Accepted 27 June 2014]
}

Background: Acrylamide (ACR) exposure is associated with neurotoxicity, carcinogenicity, and reproductive toxicity. The use of soy sauce as a condiment is common and it has been found that it possesses high antioxidant activity. The objective of the current study is to evaluate the protective role of dark soy sauce (DSS) against ACR-induced neurotoxicity in rats.

Materials and methods: Thirty-five adult male rats were divided into four groups: control, ACR given for 4 weeks, DSS given for 4 weeks before ACR, and DSS given with ACR for 4 weeks. The trigeminal ganglia and cerebellum were dissected and processed for histological staining with haematoxylin and eosin and immunohistochemistry for synaptophysin (SYP) and morphometric analysis.

Results: In the trigeminal ganglia, ACR group showed central chromatolysis, degeneration and cell loss. DSS before ACR group had less marked changes in the neuronal architecture, while in ACR with DSS group, better preservation was observed. In the cerebellum, ACR group showed shrunken Purkinje cells and nuclear pyknosis. Spacing and dissociation between Purkinje layer and other layers was seen. DSS before ACR group showed few degenerated Purkinje cells with normal pattern of the other layers of cerebellar cortex. ACR with DSS group showed less disturbed cerebellar layers architecture. Cerebellar SYP immunoexpression and its area per cent were decreased in ACR group compared with the control. It increased in both DSS treated groups, specifically DSS concomitantly given with $A C R$.

Conclusions: ACR exerted marked cellular degenerative effects and administration of DSS and ACR at the same time had neuroprotective effect. DSS treatment before ACR exposure gave only marginal improvement. (Folia Morphol 2015; 74, 1: 16-24)

Key words: dark soy sauce, acrylamide, cerebellum, trigeminal ganglion, synaptophysin

\section{INTRODUCTION}

Acrylamide (ACR) is a water-soluble vinyl monomer that has multiple chemical and industrial applications: e.g. waste water management or processing.
In addition, ACR is used extensively in molecular laboratories for gel chromatography [27]. In laboratory animals ACR exposure is associated with neurotoxicity, carcinogenicity, and reproductive toxicity, whereas

Address for correspondence: Dr H.F. Abdelall, Department of Histology, Faculty of Medicine, Cairo University, Cairo, Egypt, tel: 00966558039914, e-mail: hala2008@riyadh.edu.sa; tarekabdelmoniem@yahoo.com 
in humans, only neurotoxicity has been demonstrated for this toxicant [12]. Earlier studies indicated that subchronic, low level occupational exposure of humans and laboratory animals to ACR produced neurotoxicity characterised by ataxia, skeletal muscle weakness and numbness of the hands and feet $[4,8]$.

Recently, exposure to ACR in foodstuffs has become a worldwide concern because of its generation in a variety of fried and oven-baked foods during cooking through Maillard reactions of sugars with asparagine residues [14]. ACR initially targeted nerve terminals in both the central and peripheral nervous systems, resulting in autonomic, behavioural, sensory, and motor disturbances [6]. Early morphological studies suggested that both human and experimental neurotoxicity were mediated by cerebellar Purkinje cell injury and by degeneration of distal axons in the peripheral and central nervous system (CNS) [16]. The metabolism of ACR in the body may generate reactive oxygen species, causing the oxidative stress that may play a role in carcinogenicity [32].

The use of soy sauce as a condiment is common in the Asian diet and it has been found that much commercially available soy sauce possesses high antioxidant activity in vitro as determined by the ability to scavenge the nitrogen centred ABTS + $\left(2,2^{\prime}\right.$-azino-bis(3-ethylbenzothiazoline-6-sulfonate) radical, an assay that is frequently used to assess the antioxidant activity of beverages, food extracts, and body fluids [22]. Dark soy sauce (DSS) was especially effective in vitro [11]. In a randomised and crossover study, healthy human subjects were given DSS or placebo meals. Lower lipid peroxidation (LP) was observed in vivo in treatment with DSS [10]. Antioxidant effect of DSS in vitro or in vivo hints that DSS may play a protective role against oxidative damage induced through diet [27].

Synaptophysin (SYP) is an integral membrane glycoprotein originally isolated from presynaptic vesicles of bovine neurons. It is involved in structural functions like organising other membrane components or in targeting the vesicle to the plasma membrane [7].

The objective of the current study is to evaluate the protective action of dark soy sauce against ACR-induced neurotoxicity in rats based on histological, immunohistochemical and morphometric assessments.

\section{MATERIALS AND METHODS}

Thirty-five adult Sprague Dawley male rats, weighing 160-200 g, were maintained on standard laboratory diet and water ad libitum. The animals were treated and maintained in accordance with the guidelines for the care and use of laboratory animals in scientific research by the animal research centre, Faculty of Medicine, Cairo University. After an acclimation period of 1 week, rats were divided into the following four groups:

- Untreated control group (C): 5 rats.

- Untreated for 4 weeks then orally by gastric intubation on ACR (at concentration of $0.1 \mathrm{mg} / \mathrm{L}$ into distilled water, corresponding to $10 \mathrm{mg} / \mathrm{kg}$ body weight/day), according to an experimental protocol inducing clear neurotoxicity within 22 days in rats, for another 4 weeks [13] (A): 10 rats.

- DSS dissolved in water orally $(0.5 \mathrm{~mL} / \mathrm{kg}$ body weight/day) [27] for 4 weeks then treated orally on ACR for another 4 weeks (D-A): 10 rats.

- Untreated for 4 weeks then orally on DSS plus $A C R$ at the same time for another 4 weeks $(A+D)$ : 10 rats.

ACR was purchased from Sigma (St. Louis, MO, USA; CAS\# 79-06-1) as a white powder with a purity of $>98 \%$. Animals were housed in stainless steel cages in a temperature-controlled $\left(23 \pm 1^{\circ} \mathrm{C}\right)$ and artificially illuminated ( $12 \mathrm{~h}$ dark/light cycle) room free from any source of chemical contamination. During the animal experiment, food consumption, body weight, and water consumption were recorded weekly. All animals were euthanised by intraperitoneal injection of $120 \mathrm{mg} / \mathrm{kg}$ pentobarbital and sacrificed by exsanguinations from the abdominal aorta at the end of the experimental period.

For histological examination. The cranial base including the trigeminal ganglia was dissected and fixed in $10 \%$ buffered formalin $(\mathrm{pH} 7.4$ ) for 3 days at room temperature. After fixation, trigeminal nerves were dissected. The whole brain was dissected from the head, and then immersed in $4 \%$ sodium phosphate buffered $(0.11 \mathrm{M}, \mathrm{pH} 7.4)$ paraformaldehyde solution for 8-12 h. Then the cerebellum was dissected. All the dissected organs were routinely processed for paraffin embedding, sectioning at $3 \mu \mathrm{m}$ and staining with haematoxylin and eosin (H\&E) [2].

\section{Immunohistochemistry for SYP}

Sections obtained from buffered formalin fixed cerebellum and trigeminal ganglia were subjected to immunohistochemistry for SYP. The sections were collected on poly-L-lysine coated slides and nonspecific endogenous peroxidase activity was blocked by 

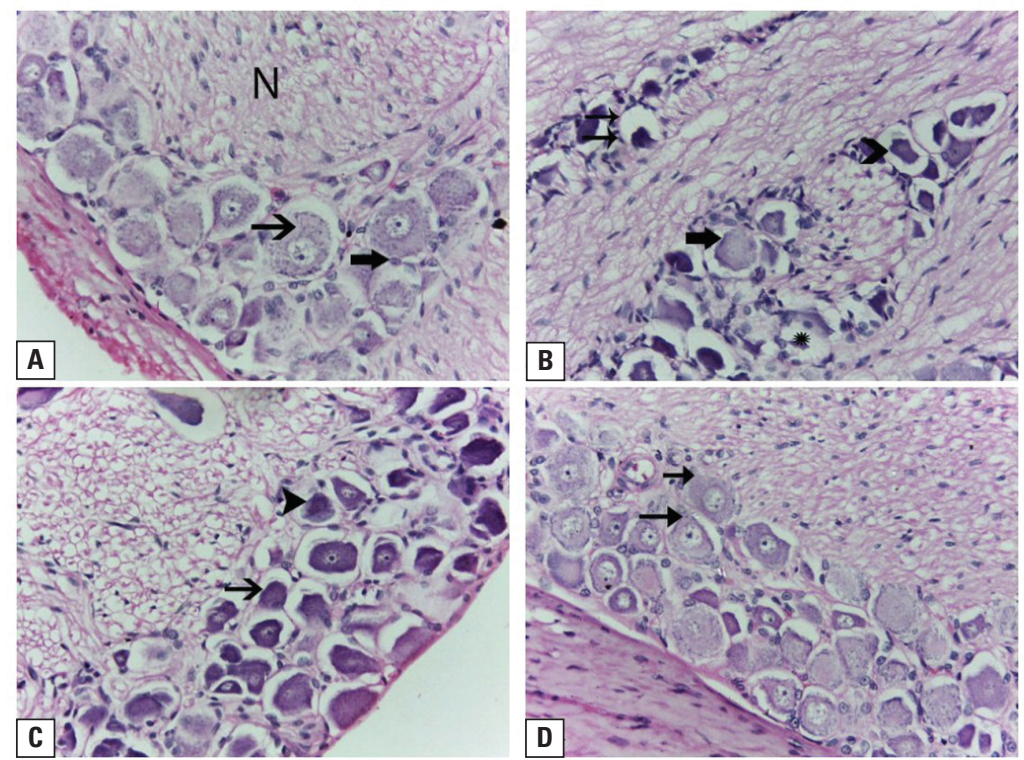

Figure 1. Photomicrographs of sections in the trigeminal ganglia of: A. Control group (group C) showing ganglion cells (thin arrow) containing a large nucleus with clear nucleolus. The cells immediately surrounding the ganglion cells as one flattened layer are satellite cells (thick arrow). Nerve fibres are visible close to or within the ganglion (N); B. Group A showing the central chromatolysis (arrows), massive degeneration of the ganglion cells (arrowhead), vacuolisation (double arrows), cell loss (asterisk); C. Group D-A showing cells that exhibited central chromatolysis (arrow) and degenerated cells (arrowhead) are still observed; D. Group A+D showing an integral ganglion cells with light cytoplasm and clear nucleus (arrow). H \& $\mathrm{E} \times 400$.

treatment with $0.9 \%$ hydrogen peroxide in absolute methanol for $10 \mathrm{~min}$. Then, they were subjected to heat pretreatment in citrate buffer. After masking with normal goat serum, sections were incubated with the primary antibody, rabbit polyclonal antibody Ab-4 (1:200, Lab Vision Corporation, Fremont, CA); overnight at $4^{\circ} \mathrm{C}$. The marker was immunohistochemically localised using $A B C$ peroxidase method where secondary polyclonal antibody formed of biotinylated anti-polyvalent antibody, streptavidin peroxidase and 3,3'-diaminobenzidine was applied. Localisation was visualised with 3,3'-diaminobenzidine and counter-stained in Meyer's haematoxylin, dehydrated, and mounted. Negative control sections were performed with the same procedure mentioned before except that the primary antibody was replaced by non-immune goat serum. Antibodies were diluted with $0.5 \%$ casein in phosphate buffered saline before application [1].

\section{Morphometric and statistical analysis}

The area per cent and optical density of SYP $(+)$ immunostaining were measured using the image analyser computer system "Lecia Qwin 500 C" (Cambridge, UK). This was done in 10 non-overlapping fields for each animal at $\times 400$. The data obtained were statistically analysed by comparing the mean values of different groups by analysis of variance ANOVA test using "SPSS 9" software. P values $<0.05$ were considered statistically significant [20].

\section{RESULTS}

\section{Histological result}

Trigeminal ganglia. Sections in control group (group C) showed large ganglion cells of pseudounipolar type with light cytoplasm containing a large oval and light hypochromic nucleus in the centre of the cell. The large cell bodies are tightly enveloped by small satellite cells. Nerve fibres are visible close to or within the ganglion (Fig. 1A). Group A showed central chromatolysis of ganglion cells. The cytoplasm became agranular and the nucleus was displaced toward the periphery of the cell. Also, massive degeneration of the ganglion cells, vacuolisation and cell loss were observed. The injured neurons were shrunken with pyknotic nuclei (Fig. 1B). Group D-A sections showed less marked changes in the neuronal architecture despite of the presence of some neurons with central chromatolysis and areas of cell loss (Fig. 1C). However, in group $A+D$, better preservation with regaining of ganglion cell and nerve fibres integrity were observed (Fig. 1D). 


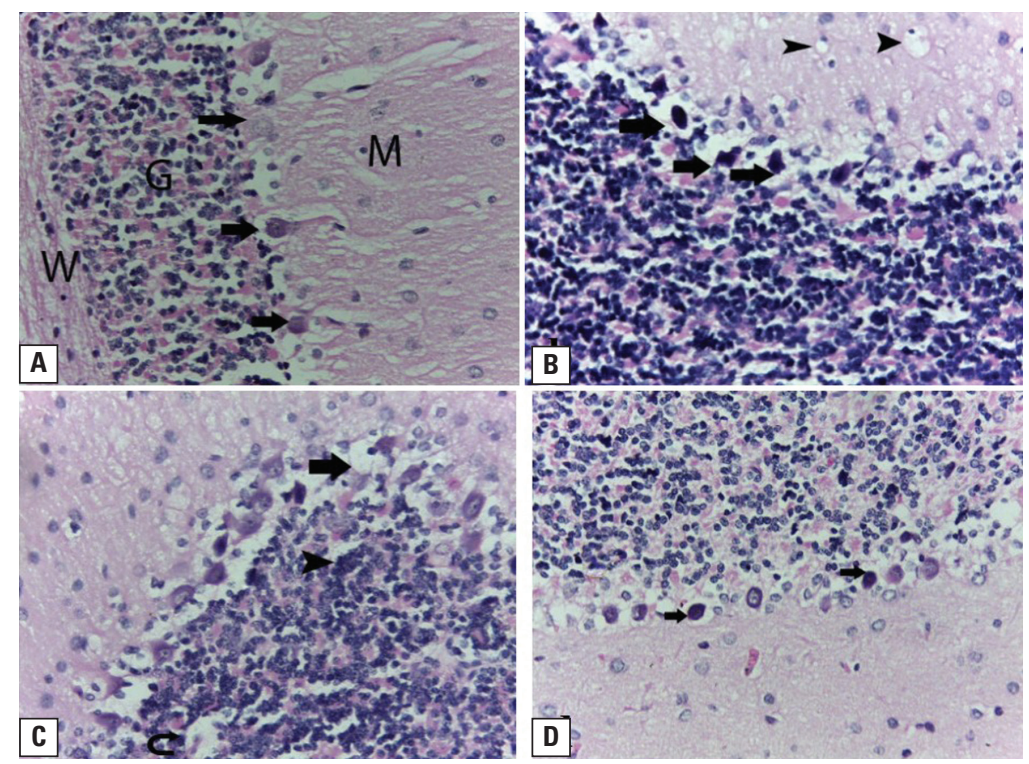

Figure 2. Photomicrographs of sections in the cerebellum of: A. Group C showing the normal architecture of cerebellum. The cortex is formed of the granular layer $(\mathrm{G})$ which is the deepest layer and consists of multiple rounded cells in groups, Purkinje cells (PCs) (arrows) appear as a single row of oval cells with large nuclei and molecular layer (M) formed of scattered rounded cells. Note the white matter (W); B. Section in the cerebellar cortex of group A rats showing shrunken irregular PCs with pyknotic nuclei surrounded by cavities (arrows). The molecular layer exhibits rounded or oval vacuoles (arrow heads); C. Section in the cerebellar cortex of group A rats showing dissociation and disorganised pattern of PCs with lysis of some cells (arrow). Hypercellularity and amalgamation of the cells of the granular layer (arrow head) and area of loss of nervous tissue (curved arrows); D. Section in the cerebellar cortex of group D-A showing some PCs are still degenerated with pyknotic nuclei (arrow).The granular and molecular layers exhibited more or less normal histological architecture. H \& E ×400.

\section{Cerebellum}

Sections of group C showed normal histological pattern of the superficial cerebellar cortex and the deep white matter. The cerebellar cortex is formed of molecular layer containing many scattered rounded cells. The granular layer was formed of multiple rounded cells that arranged in groups. The Purkinje cell (PC) layer was present between 2 layers and consisted of single row of rounded or oval cells with large nuclei (Fig. 2A). In group A PCs appeared shrunken and irregular and surrounded by empty spaces. Pyknotic nuclei or swollen nuclei were seen. For the molecular layer rounded or oval vacuoles could be detected (Fig. 2B). Some sections showed spaces and dissociation in between PCs layer and molecular layer or granular layer. Also, there was disorganised pattern and complete degeneration of PC with cell lysis. Hypercellularity of the granular layer, clumped cells and foci of necrosis were also detected (Fig. 2C). Group D-A revealed some degenerated PCs with normal pattern of the other layers of cerebellar cortex (Fig. 2D). Group A+D had less disturbed architecture of the cerebellar cortex layers which were almost similar to control sections (Fig. 3). The

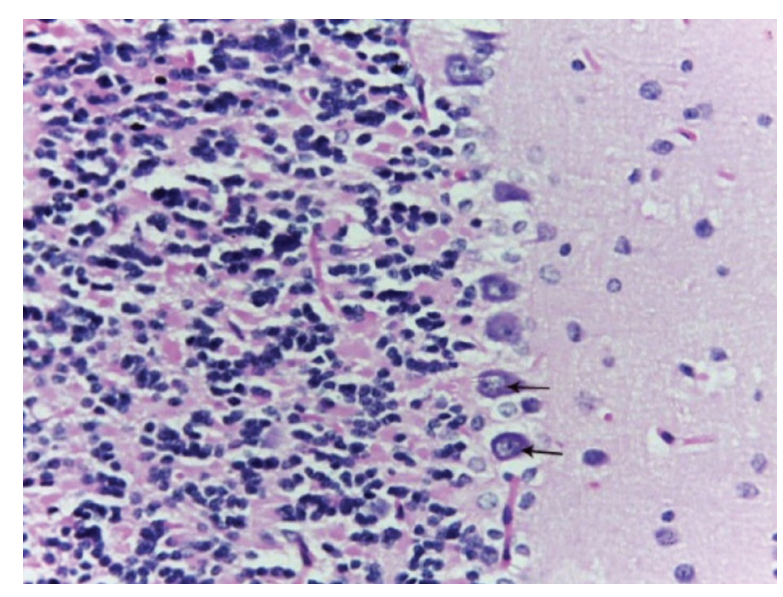

Figure 3. A photomicrograph of a section in the cerebellar cortex of group $A+D$ showing regaining of normal architecture of the cortex with integral Purkinje cells (arrows). H \& E $\times 400$.

histological characteristics of trigeminal ganglia and cerebellum of different groups were listed in Tables 1 and 2.

\section{Immunohistochemistry result}

Sections of trigeminal ganglia of group $C$ showed normal SYP immunoreactivity within the cell bodies of the ganglion cells and surrounding the 
Table 1. Histological features of the cerebellum in experimental and control groups

\begin{tabular}{lccccc}
\hline \multirow{2}{*}{ Group } & \multicolumn{2}{c}{ Purkinje layer } & Molecular layer & \multicolumn{2}{c}{ Granular layer } \\
\cline { 2 - 6 } & Degeneration & Pyknosis & Vacuolation & Hypercellularity & Necrosis \\
\hline C (control) & - & - & - & - & - \\
A & 8 & 6 & 8 & 7 & 5 \\
D-A & 5 & 3 & 4 & - & - \\
A+D & 2 & - & - & - & - \\
\hline
\end{tabular}

The number denotes number of specimens showing the finding in each group

Table 2. Histological features of the Trigeminal ganglia in experimental and control groups

\begin{tabular}{lccc}
\hline Group & \multicolumn{3}{c}{ Ganglion cell } \\
\cline { 2 - 4 } & $\begin{array}{c}\text { Central } \\
\text { chromatolysis }\end{array}$ & $\begin{array}{c}\text { Degeneration } \\
\text { and cell loss }\end{array}$ & $\begin{array}{c}\text { Pyknotic } \\
\text { nuclei }\end{array}$ \\
\hline C (control) & - & - & - \\
A & 7 & 9 & 5 \\
D-A & 3 & 4 & 2 \\
A+D & - & 1 & 1 \\
\hline
\end{tabular}

The number denotes number of specimens showing the finding in each group

nerve fibres (Fig. 4A). Faint staining is obvious in group A (Fig. 4B) which is slightly improved in group D-A (Fig. 4C) and markedly improved in group A+D (Fig. 4D). Regarding the cerebellum, in group $\mathrm{C}$, immunoreactivity for SYP as a presynaptic marker, was colocalised with the fine, often beaded labels at perisomatic regions of PCs and the brush-like processes around the PC body. In the granular layer, the SYP reactivity is not expressed within the cytoplasm of motor neurons, but forms coarsely beaded linear streaks within the neuropil which is a dense network of interwoven nerve fibres and their branches and synapses, together with glial filaments. In the molecular zone, SYP immunoreactivity was weak and diffusely distributed throughout the layer with very faint dot-like immunoreactivity (Fig. 5A). In sections that obtained from group $A$ animals, faint SYP immunoreactivity along with dendrites of PCs, granular layer and molecular layers by grading the distribution in the
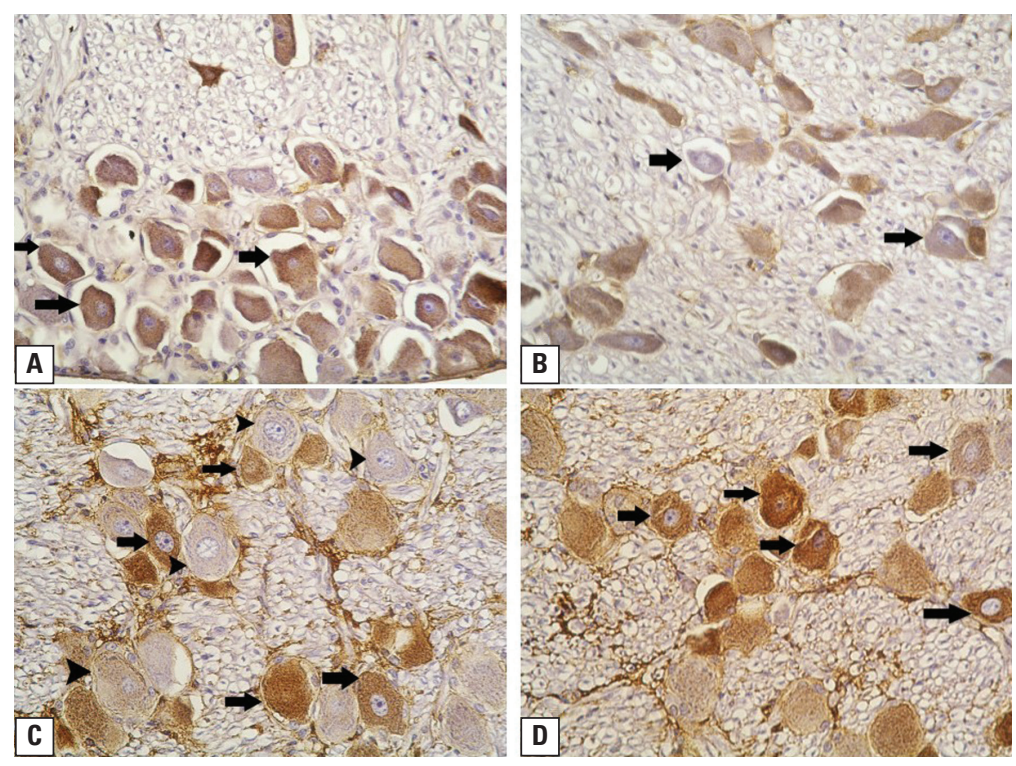

Figure 4. Photomicrographs of sections in the trigeminal ganglia of: A. Group C showing normal antibody immunostaining pattern in the form of granular cytoplasmic pattern of the ganglion neurons (arrow) and around the nerve fibres; B. Group A showing faint antibody immunostaining of the ganglion neurons (arrow); C. Group D-A showing an obvious immunoreactive cells (arrow) as well as some cells with loss of immunoreactivity (arrow head); D. Group A+D showing intense immunoreactivity of ganglion cells (arrows). Synaptophysin immunoreactivity $\times 400$. 

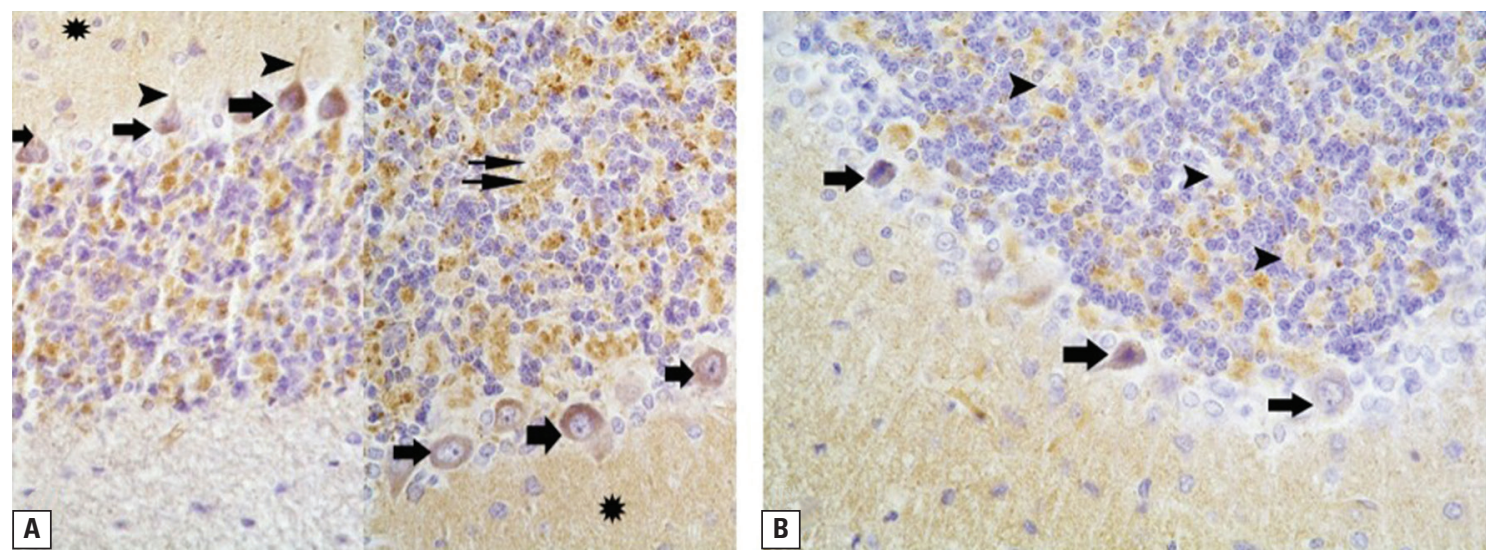

Figure 5. Photomicrographs of sections in the cerebellum of: A. Two cerebellar specimens of group C showing immunoreactivity for synaptophysin colocalised in Purkinje bodies with the fine and beaded labels and the brush-like processes (arrowheads) and around them (thick arrows). In the granular layer, the synaptophysin reactivity is forming a dense network around the cells (thin arrows). Faint immunoreaction is observed among the molecular layer (asterisk); B. Group A showing faint immunostaining of Purkinje cells (arrows) and in granular cell layer (arrow heads). Synaptophysin immunoreactivity $\times 400$.
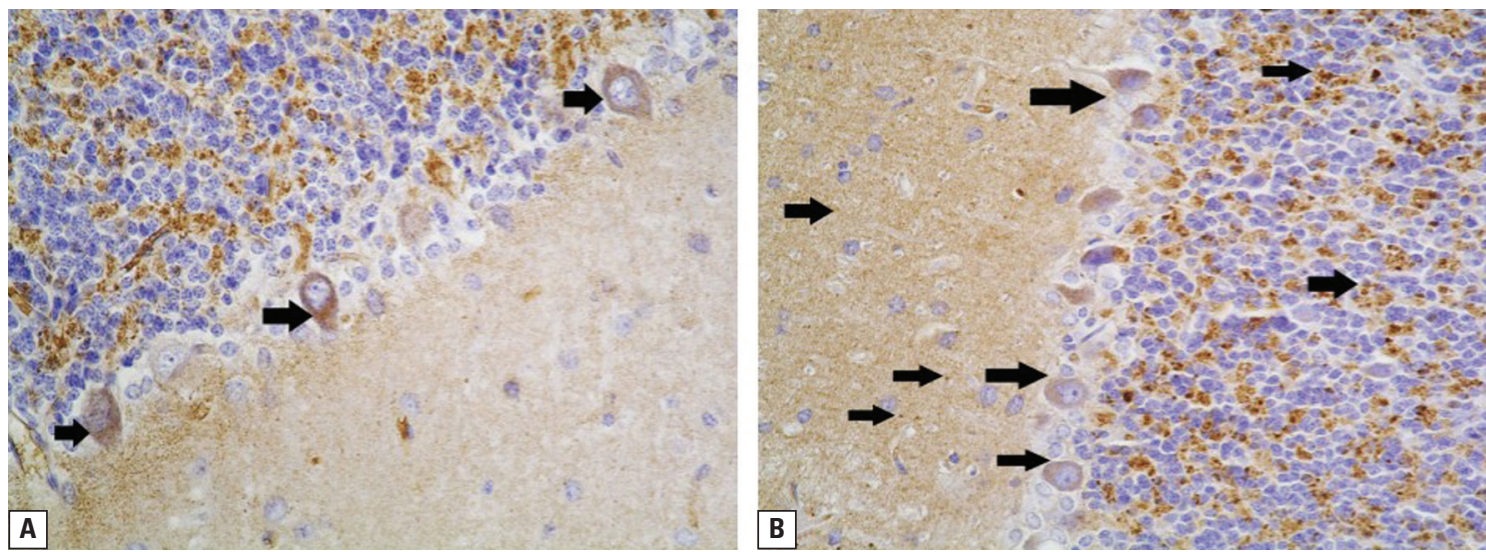

Figure 6. Photomicrographs of sections in the cerebellum of: A. Group D-A showing fair immunostaining of Purkinje cells (arrows) and the other two layers of the cerebellar cortex; B. Group A+D showing intense synaptophysin (SYP) immunoreactive in all layers of cerebellar cortex (arrows). SYP immunoreactivity $\times 400$.

full cortical area (Fig. 5B). Sections in group D-A and $A+D$ exhibited a significant intense SYP immunoreaction which was apparently clear in group $A+D$ more than group D-A (Figs. 6A, B).

\section{Morphometric results}

The area percent of SYP immunoexpression in the cerebellum significantly decreased in ACR group compared with the control. It increased in both DSS treated groups, specifically group A+D but still lower than the control values (Table 3 ). The optical density only significantly decreased in ACR group and restored to control values in both DSS treated groups (Table 4). For the trigeminal ganglion the area per cent and the optical density of SYP significantly decreased in both $A C R$ group and group $D-A$ while group $A+D$ showed nonsignificant differences from the control (Tables 5, 6).

\section{DISCUSSION}

ACR, a food contaminant, belongs to a large class of structurally similar toxic chemicals, "type-2 alkenes", to which humans are widely exposed. Occupational exposure to ACR has received wide attention through the last decades [6]. The current study was designed to investigate the ameliorating effect of DSS on ACR induced neurotoxicity and neuronal degeneration in trigeminal ganglia - as a part of peripheral nervous system - and cerebellar cortex - as a part of CNS - in rats. 
Table 3. Area per cent of synaptophysin immunoexpressionin the cerebellum per field

\begin{tabular}{lcc}
\hline & Mean \pm SD & P \\
\hline Group C (control) & $31.12 \pm 0.72$ & - \\
Group A & $16.71 \pm 0.80$ & $0.001^{*}$ \\
Group D-A & $21.32 \pm 1.38$ & $0.001^{*}$ \\
Group A+D & $26.49 \pm 1.49$ & $0.001^{*}$ \\
\hline
\end{tabular}

*Statistically significant as compared with the control $(p<0.05)$; SD - standard deviation

Table 4. Optical density of synaptophysin immunoexpressionin the cerebellum per field

\begin{tabular}{lcc}
\hline & Mean \pm SD & P \\
\hline Group C (control) & $0.85 \pm 0.1$ & - \\
Group A & $0.77 \pm 0.07$ & $0.001^{*}$ \\
Group D-A & $0.81 \pm 0.09$ & 0.098 \\
Group A+D & $0.82 \pm 0.15$ & 0.163 \\
\hline
\end{tabular}

${ }^{*}$ Statistically significant as compared with the control $(p<0.05)$; SD — standard deviation

Table 5. Area per cent of synaptophysin immunoexpression in trigeminal ganglion cells per field

\begin{tabular}{lcc}
\hline & Mean \pm SD & P \\
\hline Group C (control) & $27.23 \pm 1.84$ & - \\
Group A & $20.12 \pm 0.71$ & $0.001^{*}$ \\
Group D-A & $21.82 \pm 1.84$ & $0.001^{*}$ \\
Group A+D & $26.03 \pm 1.26$ & 0.208 \\
\hline
\end{tabular}

${ }^{*}$ Statistically significant as compared with control $(p<0.05)$; SD — standard deviation

Table 6. Optical density of synaptophysin immunoexpression in trigeminal ganglion cells per field

\begin{tabular}{lcc}
\hline & Mean \pm SD & P \\
\hline Group C (control) & $0.85 \pm 0.11$ & - \\
Group A & $0.69 \pm 0.07$ & $0.001^{*}$ \\
Group D-A & $0.71 \pm 0.06$ & $0.002^{*}$ \\
Group A+D & $0.82 \pm 0.03$ & 0.825 \\
\hline
\end{tabular}

*Statistically significant as compared with control $(p<0.05)$; SD — standard deviation

As mentioned earlier [13], the dose level of ACR of the present study was selected according to an experimental protocol inducing clear neurotoxicity within 22 days in rats. At this concentration in the present work, most animals exhibited extensive degeneration of the neurons of the ganglia and cerebellum in the form of central chromatolysis and cell lyses of the ganglion cells and shrunken PCs and foci of necrosis in the cerebellar cortex. The results are explained by previous studies $[9,19]$. They proved that ACR enhances the production of reactive oxygen species which play a role in the oxidative stress and potentially affect the brain. ACR administered rats showed increased levels of LP product, protein carbonyl content, hydroxyl radical and hydroperoxide. Free radicals are known to attack the highly unsaturated fatty acids of the cell membrane to induce LP, which is considered a key process in many pathological events and is one of the reactions induced by oxidative stress [24].

Recently, 3 important hypotheses considering ACR neurotoxicity were suggested: inhibition of kinesin-based fast axonal transport, alteration of neurotransmitter levels, and direct inhibition of neurotransmission [26]. Despite of some researchers believed that exposure of humans to relatively low levels of ACR in the diet will not result in clinical neuropathy [18], some neurotoxicologists are concerned about its cumulative neurotoxicity. It has been shown in several studies that the same neurotoxic effects can be observed at low and high doses of ACR, with the low doses simply requiring longer exposures [32].

As discussed by Seale et al. [25], in addition to signs of peripheral neuropathy that induced by ACR intoxication, some affected rats developed signs of CNS damage such as tremor, an ataxic gait, or a mild organic mental syndrome. Thus, they found that in ACR-intoxicated animals, axonal degeneration occurs in the medulla, in the anterior and lateral columns of the spinal cord, and in the nerve terminals of the cerebellum. Axonal degeneration due to ACR consumption was explained that $A C R$ interferes with the synthetic activity of the nerve cell body which gradually fails in its function to provide the neurotransmitters for the axon [28]. This leads to depletion in the amount of material reaching the distal regions of axons where degeneration begins. Another study suggested that ACR inactivates the axonal transport system by which neurotransmitters, assembled in the neuron cell body, are transported along the axon [12]. Finally, if ACR inactivated neurotransmitters in the axon, which is dependent for its supply on the nerve cell body, this alone could account for the dying-back process [27].

In the present study, extensive degeneration of the PCs of the cerebellum is prominent in ACR exposed 
group. A study which clarified that cerebellar PCs, the sole output neuron of the cerebellar cortex, is a major site of ACR action on the cerebellum [5]. Regression and degeneration of their extensive dendritic arbour occurs simultaneously with an accompanying decline in the total number of synapses/PCs.

DSS administration orally at a dose of $0.5 \mathrm{~mL} / \mathrm{kg}$ body weight/day in the current work was found to have a protective effect against ACR toxicity. Obviously, the administration of DSS plus ACR at the same time was more effective and resulted in a marked less changes of histological pattern and morphometric assessments than the DSS treatment before ACR exposure. This is in accordance with the recent study that the inhibition of LP by DSS is attributable to its free radical scavenging activity [31]. In this regard, in vitro or in vivo studies show that DSS possesses high antioxidant activity as determined by the ability to scavenge the nitrogen cantered ABTS + radical and decrease LP [10, 11, 22].

Shuming et al. [27] proved the protective role of DSS. They declared that serotonin is known as a neurotransmitter and it synthesised in the intestinal chromaffin cells, central and peripheral nervous systems. They proved that animals treated with ACR showed a significant decrease in serum serotonin; this decrease was weakened in ACR combined DSS rats. Based on hormonal assays, they also proved that there was a decrease in plasma adrenaline indicating the degenerative effects of $A C R$ on both brain and the chromaffin cells in the adrenal medulla. These findings supported the earlier finding [12]. The combined treatments of ACR with soy sauce were effective in the elevation of the decreased level of this hormone.

Upon biochemical analysis, 3-hydroxy-2-methyl-4H-pyran-4-one (maltol) was one of several active compounds found in an ethyl acetate extract of DSS and was present at millimolar concentrations. Carbohydrate containing pigments such as melanoidins were present in coloured fractions of DSS. Both were suggested to be the major contributors to the high antioxidant capacity of DSS [30].

In the present study, SYP immunoexpression in both trigeminal ganglia and cerebellum, showed faint staining in ACR exposed group and markedly increased in DSS treated groups. This was also confirmed in the morphometric results. The function of this protein is still unknown, but previous suggestions have included calcium binding [23], channel formation [29], exocytosis [17], and synaptic vesicle recycling via endocytosis [7]. SYP, an integral membrane protein of synaptic vesicles, is involved in several crucial aspects of synaptic vesicle trafficking, including the initiation of neurotransmitter release [23]. Moreover, previous work showed that SYP plays an important regulatory role in activity-dependent competitive synapse formation in cultures of hippocampal neurons [29].

Normally in the granular layer, the SYP reactivity is not expressed within the cytoplasm of motor neurons, but forms coarsely beaded linear streaks within the neuropil which is a dense network of interwoven nerve fibres and their branches and synapses, together with glial filaments. This activity probably represents presynaptic vesicles within terminal axons as confirmed by Pyeon and Lee [21]. Recently it is confirmed that since the SYP is a protein localised in pre-synaptic vesicles, so it can be used as a reliable marker for synaptic covering evaluation. Their findings were concomitant with the current study findings. They demonstrated that an increase in SYP expression as well as the number of synaptic vesicles in the cerebellum and hippocampus whereas the treatment will strengthen the synaptic contacts decreasing the loss of inputs to injured motor neurons [15]. Accordingly, in the present study DSS treated group maintained more elevated immunoreactivity against SYP indicating a better synaptic preservation.

In accordance with the present findings, Barbizan et al. [3] showed that SYP immunoexpression significantly decreased in sciatic motor nuclei after ventral root avulsion. There was a reduction of presynaptic terminals at the microenvironment of the injured motor neurons. This was in contrast to the ventral root avulsion and reimplantation. The repair of axonal injury resulted in preservation of SYP immunoreactivity, especially in the immediate vicinity of the motor neurons.

However, other researchers had different findings. The result of the current study is controversial to what was reported by other researchers [31]. They claimed that numbers of SYP $(+)$ aberrant dots per $m m$ cortex in the cerebella molecular layer, significantly increased in the ACR group compared with the control and decreased in DSS treated groups. They suggested that SYP aberrant dots indicated a nerve terminal injury of the CNS after ACR exposure and DSS had a protective effect by decreasing the number of the aberrant dots. 


\section{CONCLUSIONS}

ACR exerted marked cellular degenerative effects and administration of DSS and ACR at the same time had neuroprotective effect. DSS treatment before ACR exposure gave only marginal improvement.

\section{REFERENCES}

1. Bancroft JD, Cook HC (1994) Manual of histological techniques and diagnostic applications. Churchill Livingstone, Edinburgh, pp. 263-325.

2. Bancroft JD, Stevens A (1996) Theory and practice of histological techniques. $4^{\text {th }}$ Ed. Churchill Livingstone, London UK, p. 104.

3. Barbizan R, Castro MV, Rodrigues AC, Barraviera B, Ferreira RS, Oliveira AL (2013) Motor recovery and synaptic preservation after ventral root avulsion and repair with a fibrin sealant derived from snake venom. PLOS One, 8: e63260.

4. Deng H, He F, Zhang S, Calleman CJ, Costa LG (1993) Quantitative measurements of vibration threshold in healthy adults and acrylamide workers. Int Arch Occup Envirom Health, 65: 53-56.

5. Dlugos C (2008) Ethanol-related increases in degenerating bodies in the purkinje neuron dendrites of aging rats. Brain Res, 1221: 98-107.

6. Erkekoglu P, Baydar T (2013) Acrylamide neurotoxicity. Nutr Neurosci, 27: 49-57.

7. Evans G, Cousin M (2005) Tyrosine phosphorylation of synaptophysin in synaptic vesicle recycling. Biochem Soc Trans, 33: 1350-1353.

8. He F, Zhang S, Wang H (1989) Neurological and electroneuromyographic assessment of the adverse effects of acrylamide on occupationally exposed workers. Scand J Work Environ Health, 15: 125-129.

9. Lakshmi D, Gopinath K, Jayanthy G, Anjum S, Prakash D, Sudhandiran G (2012) Ameliorating effect of fish oil on acrylamide induced oxidative stress and neuronal apoptosis in cerebral cortex. Neurochem Res, 37: 1859-1867.

10. Lee $C Y$, Isaac $H B$, Wang $H$, Huang $H$, Long $L H$, Jenner AM, Halliwell B (2006) Cautions in the use of biomarkers of oxidative damage; the vascular and antioxidant effects of dark soy sauce in humans. Biochem Biophys Res Commun, 344: 906-911.

11. Long LH, Halliwell B (2001) Antioxidant and prooxidant abilities of foods and beverages. Methods Enzymol, 335: 181-190.

12. LoPachin RM (2004) The changing view of acrylamide neurotoxicity. Neurotoxicology, 25: 617-630.

13. LoPachin RM, Castiglia CM, Saubermann AJ (1992) Acrylamide disrupts elemental composition and water content of rat tibial nerve. Toxicol Appl Pharmacol, 115: 21-34.

14. Ma Y, Shi J, Zheng M, Liu J, Tian S, He X, Zhang D, Li G, Zhu J (2011) Toxicological effects of acrylamide on the reproductive system of weaning male rats. Toxicol Ind Health, 27: 617-627.

15. Matheus P, Suzana U, Luciana P, Cartarozzi E, Francisco S, Alexandre L (2013) Neuroprotection and reduction of glial reaction by cannabidiol treatment after sciatic nerve transection in neonatal rats. Eur J Neuroscience, 38: 3424-3434.
16. Miwa T, Makoto S, kaoru I, Hitoshi F, Masao H, Akiyoshi N (2008) Pathological assessment of the nervous and male reproductive system of rat offspring exposed maternally to acrylamide during the gestation and lactation periods: a preliminary study. J Toxicol Sciences, 33: 11-24.

17. Mullany PM, Lynch MA (1998) Evidence for a role for synaptophysin in expression of long-term potentiation in rat dentate gyrus. Neuro Report, 9: 2489-2494.

18. Nakagawa-Yagi Y, Choi DK, Ogane N, Shimada S, Seya M, Momoi T, Ito T, Sakaki Y (2001) Discovery of a novel compound: insight into mechanisms for acrylamide-induced axonopathy and colchicine-induced apoptotic neuronal cell death. Brain Res., 909: 8-19.

19. Patel HC, Boutin H, Allan SM (2003) Interleukin-1 in the brain: mechanisms of action in acute neurodegeneration. Ann NY Acad Sci, 992: 39-47.

20. Petrie A, Sabin C (2005) Medical Statistics at a Glance. 2nd Ed. In: Sugden M, Moore K eds. Blackwell Publishing Ltd., USA, p. 55.

21. Pyeon HJ, Lee YI (2012) Differential expression levels of synaptophysin through developmental stages in hippocampal region of mouse brain. Anat Cell Biol, 45: 97-102.

22. Re R, Pellegrini N, Pannala A, Yang M, Rice-Evans C (1999) Antioxidant activity applying an improved ABTS radical cation decolorization assay. Free Radic Biol Med, 26: 1231-1237.

23. Rehm H, Wiedenmann B, Betz H (1986) Molecular characterization of synaptophysin, a major calcium-binding protein of the synaptic vesicle membrane. EMBO J, 5 : 535-541.

24. Schinella GR, Tournier HA, Prieto JM, Mordujovich de Buschiazzo P, Ríos JL (2002) Antioxidant activity of anti-inflammatory plant extracts. Life Sci,70: 1023-1033.

25. Seale SM, Feng Q, Agarwal AK, El-Alfy AT (2012) Neurobehavioral and transcriptional effects of acrylamide in juvenile rats. Pharmacol Biochem Behav, 101: 77-84.

26. Shinomol GK, Raghunath N, Bharath MM, Muralidhara M (2013) Prophylaxis with Bacopa monnieri attenuates acrylamide induced neurotoxicity and oxidative damage via elevated antioxidant function. Cent Nerv Syst Agents Med Chem, 13: 3-12.

27. Shuming C, Jilin F, Xichun Z (2009) The moderating role of dark soy sauce to acrylamide-induced oxidative stress and neurophysiological perturbations in rats. Toxicol Mechanism Methods, 19: 434-440.

28. Stone JD, Peterson AP, Eyer J., Oblak TG, Sickles DW (2001) Neurofilaments are nonessential to the pathogenesis of toxicant induced axonal degeneration. J Neuroscience, 21: 2278-2287.

29. Sudhof TC, Lottspeich F, Greengard P, Mehl E, Jahn R (1987) A synaptic vesicle protein with a novel cytoplasmic domain and four transmembrane regions. Science, 238: 1142-1144.

30. Wang H, Jenner AM, Lee CY, Shui G, Tang SY, Whiteman M, Wenk MR, Halliwell B (2007) The identification of antioxidants in dark soy sauce. Free Radic Res, 41: 479-488.

31. Xichun Z, Min'ai Z (2009) Protective role of dark soy sauce against acrylamide induced neurotoxicity in rats by antioxidative activity. Toxicol Mechanisms Methods, 19: 369-374.

32. Yousef MI, El-Demerdash FM (2006) Acrylamide-induced oxidative stress and biochemical perturbations in rats. Toxicology, 219: 133-141. 